Focussed on "Crack Paths"

\title{
Size effect on the fracture resistance of rough and frictional cracks
}

\author{
Andrea Spagnoli, Andrea Carpinteri, Michele Terzano \\ Department of Engineering and Architecture, University of Parma, Parco Area delle Scienze 181/A, 43124 Parma, Italy \\ spagnoli@unipr.it
}

\begin{abstract}
Elastic fracture mechanics commonly defines the fracture resistance of brittle materials within an idealized picture of planar and traction-free cracks. An efficient approach to describe the interface conditions in real cracks, such as those occurring in concrete, ceramics or stones, is to include the effect of both roughness and friction by means of a constitutive relationship between opposite points on the interface. In the present paper, we use a numerical technique, based on the solution of singular integral equations, to derive the near-tip stress field with various interface conditions. Then, the technique is applied to investigate the size effect of the interface roughness, where such an effect is related to the ratio between the characteristic length of the roughness and the nominal length of the crack. It is found that the resulting near-tip stresses can be profoundly influenced by the crack path, particularly for short cracks.
\end{abstract}

KEYwORDS. Interface models; Friction; Roughness; Distributed dislocation technique; Size effect.
Citation: Spagnoli, A., Carpinteri, A., Terzano, M., N., Size effect on the fracture resistance of rough and frictional cracks, Frattura ed Integrità Strutturale, 47 (2019) 401-407.

Received: 26.10 .2018

Accepted: 08.11.2018

Published: 01.01.2018

Copyright: (C) 2019 This is an open access article under the terms of the CC-BY 4.0, which permits unrestricted use, distribution, and reproduction in any medium, provided the original author and source are credited.

\section{INTRODUCTION}

I $\mathrm{n}$ linear elastic fracture mechanics (LEFM), the stress field in proximity of the tip of a crack is described by a single parameter, the stress intensity factor (SIF), which is consequently used to give a measure of the fracture resistance of a certain material. Such an approach is based on the assumption of small-scale yielding, and considers a crack with flat and smooth interfaces, neglecting any interaction between its surfaces. This rather idealised picture might be satisfactory for mode I crack growth, because the surface interaction is somehow limited; however, when the fracture surfaces are displaced relative to one another in shear, the interaction cannot be neglected.

Observations on the failure of quasi-brittle and brittle materials, such as concrete, ceramics, rocks or glass, revealed that crack growth does not proceed planarly, but on the contrary tortuous topologies of the crack paths are noticed. A combination of different factors is used to explain the observed behaviour, including the effect of far field multi-axial stresses, residual stresses, microstructural inhomogeneities, and material properties dispersion [1-5]. In many polycrystalline and aggregate materials, the crack surfaces are not macroscopically flat, instead they consist of several asperities, with peculiar size, which interact in a complex combination of sliding, sticking, climbing and deforming [6]. As a consequence, the fracture strength can be strongly altered, particularly in the presence of mixed-mode loading as a result 
of far field stresses, or geometrical and material discontinuities. Specifically, Ballarini and Plesha [7] noticed that a pure mode I crack growth occurs in very few cases, since the mode mixity is often a result of the crack tortuosity.

The same Authors presented an interface model, previously proposed by Plesha [8], to deal with the effect of roughness and friction along the crack surfaces in a simplified way, and included such a model in a numerical algorithm based on the solution of singular integral equations. The same effect, appropriately defined 'sliding mode crack closure', was observed by Tong et al. [9] in the analysis of cracks under shear fatigue loading. On the other hand, the interaction of the crack surfaces through the asperities generates a mode I component, even if the remote loading is purely mode II. This phenomenon is generally known as dilatancy, that is, an opening displacement caused by the coupling between normal and tangential directions along the nominal crack line.

In the present paper, we adopt an interface model to account for the effects of friction and roughness, which follows from the work of Plesha [8], and whose complete formulation has been firstly presented by the authors in [10]. Frictional effects are described with the classical Coulomb's law, while the roughness is exemplified by a uniform distribution of rigid saw-tooth asperities. The solution technique follows an analytical method derived from the application of the complex function theory, generally known as the Distributed Dislocation Technique (DDT), which has been applied successfully in the solution of crack problems with different geometric configurations [11]. We have previously showed its potential in characterising the near-tip stress fields of cracks under mixed mode loading, specifically the effect of the interface interaction with respect to the onset of crack propagation under monotonic loading [12].

The aim of this study is to further explore the effects of interface interaction, by exploring the possible influence on the fracture resistance of a size effect, introduced by a characteristic length of the material roughness. It is well known that the strength of materials, both the fracture resistance and the fatigue limit, is generally affected by the size of the specimen or its microstructural properties. Studies have tried to explain this phenomenon by adopting a fractal description of the microstructure of brittle and disordered materials, and noticed that the fatigue crack growth rate depends not only on a fractal dimension but also, in an explicit fashion, on the crack length [13-16]. For sinusoidally-patterned surfaces, the size effect on the critical load has been related to the ratio between the amplitude and the wave length [17]. In this work, the size effect is related to the ratio between the crack roughness period and the length of the crack. Specifically, we explore the influence of both the height and the length of the saw-tooth asperities on the mode II stress intensity factor, under a mixed-mode loading condition. The paper is structured in two main sections. We start with a Formulation section where we review the interface model and give some details on the numerical algorithm that we have used to compute the stress intensity factor; following, we present the Results, obtained by an application of the method to a simple edge-cracked geometry, and discuss the implications of the interface asperities and the size effect.

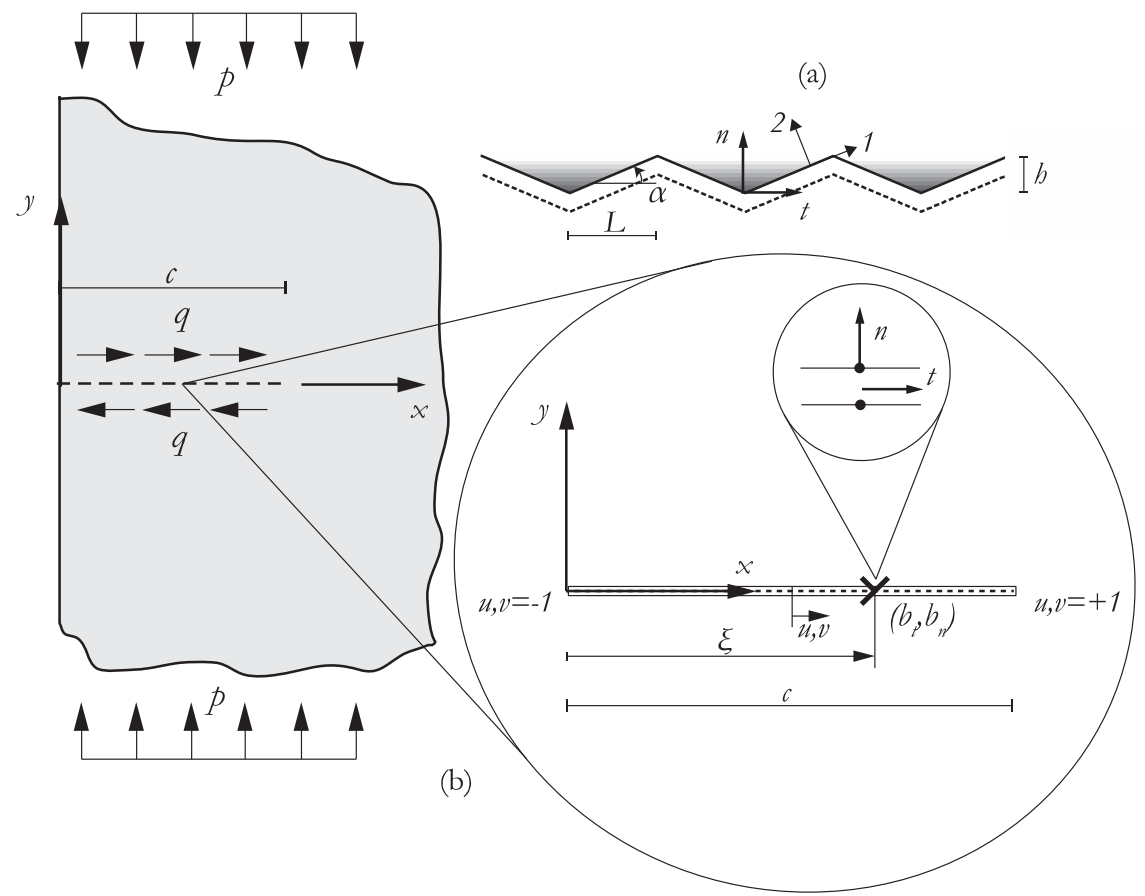

Figure 1: a) The saw-tooth asperity model, showing the local and global reference systems adopted in the formulation, Eqns. (1)-(4). b) Schematic model of the geometry with an edge crack of length $c$. 


\section{FORMULATION}

\section{Description of the model}

T $\mathrm{n}$ order to account for the effects of friction and roughness, we make use of an interface model formulated as a constitutive relationship between opposing points along the crack, written in the form of a classic elastic-plastic law of small strain plasticity. Globally, the crack is smooth and frictionless and the surface interference is modelled by means of bridging stresses between the crack surfaces. In this fashion, we can obtain a straightforward implementation within the distributed dislocation technique, as the bridging stresses, computed at a discrete number of points, are simply added to the stress distribution resulting from the remote loads.

Let us define the relative displacement (increments) between two opposing points of the crack as composed of a recoverable elastic part $d w_{i}^{E}$, related to the remote stress field, and a non-recoverable plastic part $d w_{i}^{P}$, which accounts for frictional sliding and dilatancy. The stresses on the crack interface are related to the displacement increments by means of interface stiffnesses $E_{i j} E P$ :

$$
d \sigma_{i}=E_{i j}^{E P} d w_{j} \quad i, j=t, n
$$

where $t, n$ denote, respectively, the tangential and normal directions with respect to the nominally flat crack surface (Fig. 1a). In order to compute the interface stiffness, we need to introduce a slip function $F$ and a slip potential $G$. It can be noticed that, since Coulomb's friction law is non-associated, $F$ and $G$ do not coincide, and the direction of slip is given by the gradient of $G$. Therefore, we can express the non-recoverable displacement increment as:

$$
d w_{i}^{p}=0 \quad \text { if } \mathrm{F}<0 \text { or } \mathrm{dF}=0 \quad d w w_{i}^{p}=\lambda \frac{\partial G}{\partial \sigma_{i}} \quad \text { if } \mathrm{F}=\mathrm{dF}=0
$$

while the interface stiffness $E_{i j}^{E P}$ is computed according to the following equation:

$$
E_{i j}^{E P}=E \quad \text { if } \mathrm{F}<0 \text { or } \mathrm{dF}=0 \quad E_{i j}^{E P}=E_{i j}-\frac{\frac{\partial F}{\partial \sigma_{p}} E_{i q} E_{p j} \frac{\partial G}{\partial \sigma_{q}}}{\frac{\partial F}{\partial \sigma_{p}} E_{p q} \frac{\partial G}{\partial \sigma_{q}}} \quad \text { if } \mathrm{F}=\mathrm{dF}=0
$$

$E_{i j}$ is the elastic interface stiffness, which is taken two to four orders of magnitude greater than the elastic modulus of the medium itself, in order to assure the impenetrability between the crack surfaces and ensure good numerical compatibility. The surface roughness is described through a saw-tooth model, characterised by a constant angle $\alpha$, a mean length of the asperities equal to $2 L$ and a height $h$. The coefficient of Coulomb's friction $f$ is kept constant. With the previous assumptions, we obtain the following formulation of $F$ and $G$ :

$$
\begin{aligned}
& F=\left|\sigma_{1}\right|+f \sigma_{2}=\left|\sigma_{n} \sin \alpha+\sigma_{t} \cos \alpha\right|+f\left(\sigma_{n} \cos \alpha-\sigma_{t} \sin \alpha\right) \\
& G=\left|\sigma_{1}\right|=\left|\sigma_{n} \sin \alpha+\sigma_{t} \cos \alpha\right|
\end{aligned}
$$

We can define a crack size parameter by considering the ratio $c / L$, where ideally $c / 2 L$ approaching the unity identifies the case of a short crack. The parameters used to describe the surface roughness are somehow related to the specific material, and the scale length might differ of several orders of magnitude. For instance, the fracture process in concrete is influenced by particles and voids whose sizes are in the order of few nanometres, but if the aggregate is particularly coarse the size of the irregularities can go up to some millimetres [18]. It is also common to evaluate roughness through a digitalisation of the crack profiles, and consider it as a high-frequency shortwave length component of the measured surface, characterised through average measures or peak-to-valley heights [19].

\section{The numerical algorithm}

In this Sub-Section, we briefly summarise the numerical algorithm used in the present work, which is based on the application of the distributed dislocation technique, combined with the interface model introduced above. We begin by 
introducing a suitable distribution of dislocations along the crack surface: dislocations are commonly used in the theory of elasticity as kernel of integral equations, to describe the singular stress state occurring near a source of discontinuity, such as the tip of a crack. The stresses thereby obtained, known as the corrective term, assume the following formulation (refer to Fig.1b for an explanation of the variables):

$$
\bar{\sigma}_{i}(x)=\frac{2 \mu}{\pi(\kappa+1)} \int_{0}^{c}\left(\frac{B_{i}(\xi)}{x-\xi}+B_{j}(\xi) F_{i j}(x, \xi) d \xi\right)
$$

where $\mu$ is the elastic shear modulus and $\kappa$ is the Kolosov constant of the material. $F_{i j}$ are influence functions, whose expression for the case considered here can be found in the literature [11] and $B_{i}(\xi)$ is the dislocation density, which yields the relative displacement between the crack surfaces by integration. Applying the superposition principle, we obtain the stress state along the crack surface, adding the stresses generated by the remote loads $\sigma^{\infty}(x)$ to the corrective term in (5). If we assumed a traction-free crack, the overall stress state on the surfaces would have to be null; here, on the contrary, we need to add the bridging stresses $\sigma^{b}(x)$ resulting from the surface interaction, so that the integral formulation takes the following form:

$$
\sigma^{\infty}(x)+\frac{2 \mu}{\pi(\kappa+1)} \int_{0}^{c}\left(\frac{B_{i}(\xi)}{x-\xi}+B_{j}(\xi) F_{i j}(x, \xi) d \xi\right)=\sigma^{b}(x)
$$

The standard method of solution for integral equations of this kind consists in normalising the interval between [-1,1], so that the variables $x, \xi$ are replaced by $u$ and $v$. We can also express the unknown dislocation densities as follows:

$$
B_{j}(u)=\phi_{j}(u) \omega(u)=\phi_{j}(u) \sqrt{\frac{1+u}{1-u}}, \quad j=x, y
$$

where $\omega(u)$ is the fundamental singular function while $\phi_{j}(u)$ are the unknowns. Here we have assumed the dislocation densities to be square root singular at the tip of the crack $(u=+1)$ and bounded to zero at the crack mouth $(u=-1)$. Using Gauss-Chebyshev numerical quadrature, the integral Eqn. (6) is converted in a set of non-linear algebraic equations:

$$
\frac{2 \mu}{\pi(\kappa+1)} \sum_{k=1}^{N} W\left(u_{k}\right)\left[\frac{\phi_{i}\left(u_{k}\right)}{v_{l}-u_{k}}+\phi_{j}\left(u_{k}\right) F_{i j}\left(u_{k}, v_{l}\right)\right]=-\sigma_{i}^{\infty}\left(v_{l}\right)+\sigma_{i}^{b}\left(v_{l}\right)
$$

where $W\left(u_{k}\right)$ are weight functions. We recall that the integration points $u_{k}$ are the points at which the displacements are computed, whereas the collocation points $v_{l}$ are those at which we evaluate the stresses. In the usual applications of the dislocation methods, the right-side of Eqn. (8) is known, often null in the case of open cracks or of separating contacts, so that the system of algebraic equations is linear. Instead, in our case the bridging stresses are function of the relative displacements, through the constitutive relationship of Eqns. (1)-(5). The displacements can be obtained from the dislocation densities through the following integration:

$$
w_{i}(u)=\int_{u}^{1} B_{i}(u) d u
$$

The resulting system is therefore non-linear. An efficient technique of solution is achieved if we introduce a compliance matrix, which directly connects stresses and displacements so that we eliminate the need to integrate the dislocation densities at each step of the incremental solution. For each increment of the external loads, the stiffness matrix $E_{i j}{ }^{E P}$ in (5) needs to be updated, using the configuration of stresses and displacements at the beginning of the increment. Details of the technique are provided in [12].

The stress intensity factors at the crack tip are computed from the unknown functions $\phi(j)$, through an extrapolation to the singular point $u=+1$ : 


$$
K_{I}=\frac{2 \mu}{\pi(\kappa+1)} \sqrt{2 \pi c} \phi_{n}(+1), \quad K_{I I}=\frac{2 \mu}{\pi(\kappa+1)} \sqrt{2 \pi c} \phi_{t}(+1)
$$

\section{RESULTS}

I $\mathrm{n}$ this Section we summarise the results that we have obtained, applying the method to a simple geometry, consisting of an edge crack in a half-plane. The material is linear elastic and a state of plain strain is assumed. Compressive and tangential stresses are applied remotely, such that uniform normal and shear stresses, $p$ and $q$ respectively, act along the crack line (see Fig. 1b). In order to explore the influence of the roughness ratio, we have performed several analyses, varying the ratio $c / L$ and computed the mode II SIF at the crack tip. All the results presented in this Section are normalised with respect to the crack length $c$ and the mean normal pressure $p$.

In Fig.2 we show the variation of the normalised SIF during the increment of external loads, considering two different values of the asperity angle $\alpha$. We observe that $K_{I I}$ is null until the stress ratio $q / p$ reaches 0.5 , which is the value of the coefficient of friction $f$ chosen in the simulation: this was expected, since the stress distribution due to the external loads is constant. For $q / p>0.5$, we notice an increase, which is more pronounced for shorter cracks, i.e. for $c / L$ tending to 1.

In Fig. 3 we show the variation of the SIF, evaluated at $q / p=1$, with the angle of roughness, for three different values of $c / L$. We can appreciate the effect of the roughness angle, which causes a decrease of the mode II SIF: as already noticed in [12],the reason for this is that the surface roughness generates a resistance to sliding, which is added to the one due to friction. However, while the decrease is almost linear for short cracks, for bigger $c / L$ ratios we observe a greater reduction, although it appears to attenuate at bigger roughness angles.
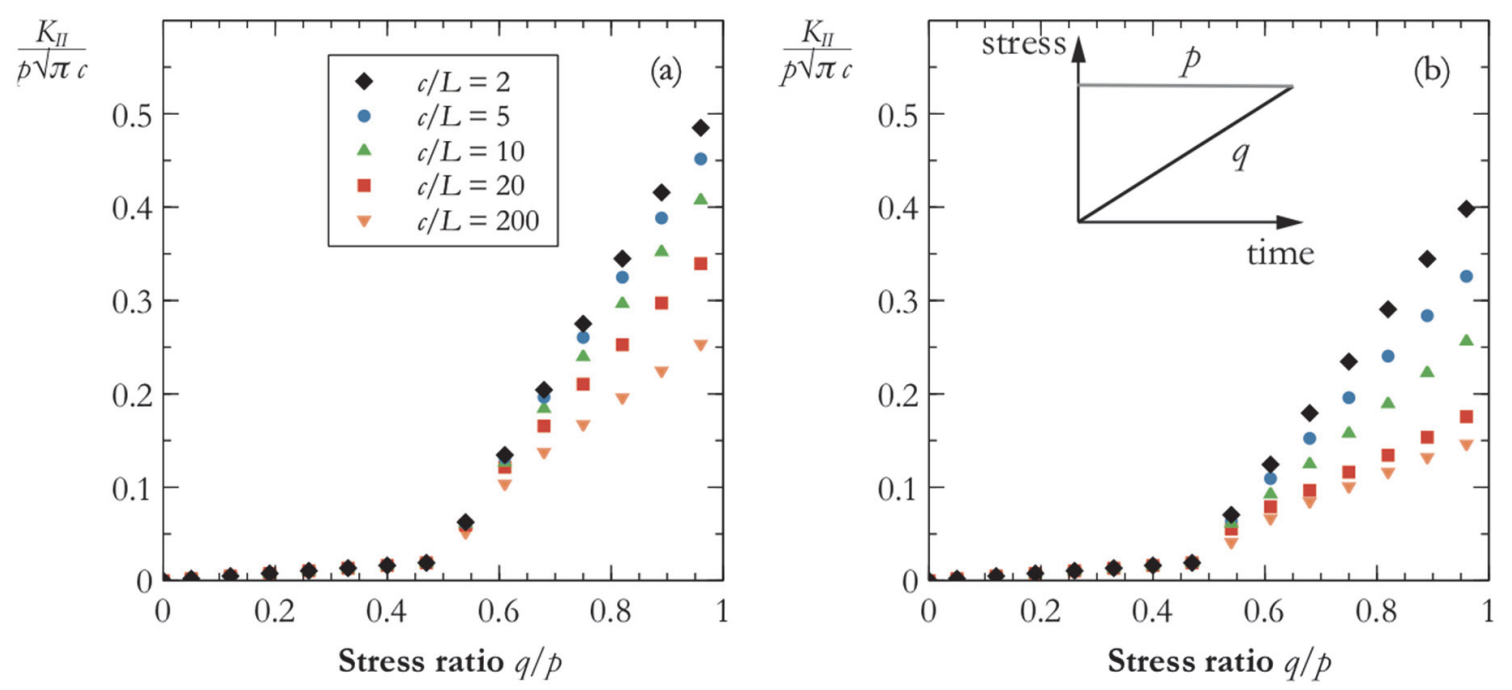

Figure 2: The normalised stress intensity factor $K_{I I}$ versus the applied load ratio $q / p$. Different relative crack lengths $c / L$ are considered. $f=0.5$. (a) Angle of the surface asperity $\alpha=2^{\circ}$ and (b) angle of the surface asperity $\alpha=5^{\circ}$. The inset shows the loading history.

Finally, we explore the effect of the ratio $c / L$, considering two different cases: in Fig.4a, we keep the angle $\alpha$ constant, while in Fig.4b we keep constant the height $b$ of the asperities. Indeed, throughout the other simulations, we have always kept the asperity angle constant: as a consequence, a change in the ratio $c / L$ implies also a change in the height $b$ of the saw-tooth.

\section{CONCLUSIONS}

7 he crack tip stress fields in brittle and quasi-brittle material can be highly influenced by the surface interaction due to microscopic asperities. As a result, the stress intensity factor might differ from the theoretical values computed according to the relationships of LEFM, which assumes a crack with flat and smooth surfaces. 
In the present paper a numerical algorithm, based on the distributed dislocation technique, is used in combination with a non-linear interface model to characterise the near-tip stress field of rough and frictional cracks. The interface roughness and the frictional effects are included through a rigid-plastic constitutive relationship between stresses and relative displacements along the crack. The stress intensity factors are computed through a numerical solution of singular integral equations, adopting an iterative algorithm developed by the authors, which has proved to be effective in different geometries and loading scenarios.

Specifically, the model is used here to examine the influence of a size effect parameter, related to the ratio between the crack roughness period and the length of the crack. The results confirm a crack shielding effect due to roughness and friction, that is, a reduction in the mode II stress intensity factor for increasing roughness (notably, the asperity angle). Moreover, we show that the crack shielding is more pronounced for longer cracks.

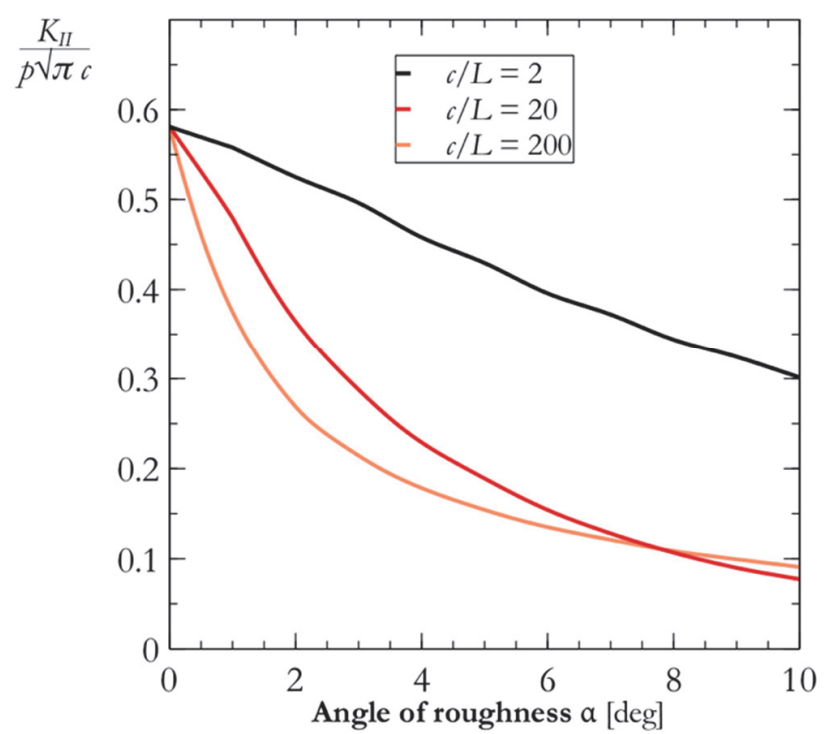

Figure 3: Variation of the normalised SIF $K_{I I}$ with respect to the roughness angle, for $q / p=1$.
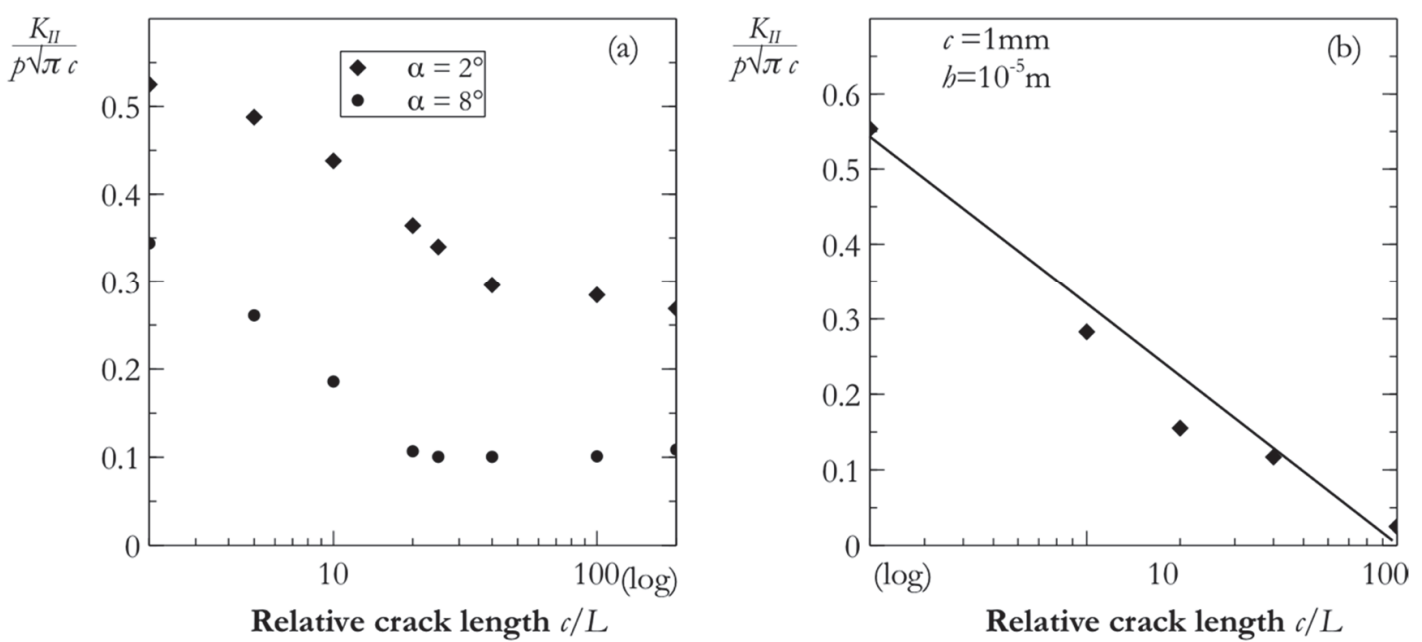

Figure 4: (a) Variation of the normalised SIF $K_{I I}$ with respect to the relative crack length $c / L$, for $q / p=1$. The angle of the surface asperity is kept constant. (b) Variation of the normalised SIF $K_{I I}$ with respect to the relative crack length $c / L$, for $q / p=1$. The height of the surface asperity is kept constant. The coefficient of friction is equal to 0.5 in all the simulations. 


\section{REFERENCES}

[1] Kitagawa, H., Yuuki, R., and Ohira, T. (1975). Crack-morphological aspects in fracture mechanics, Eng. Fract. Mech., 7(3), pp. 515-529, DOI: 10.1016/0013-7944(75)90052-1.

[2] Suresh, S. (1983). Crack deflection: Implications for the growth of long and short fatigue cracks, Metall. Trans. A, 14(11), pp. 2375-2385, DOI: 10.1007/BF02663313.

[3] Evans, A.G. and Hutchinson, J.W. (1989). Effects of non-planarity on the mixed mode fracture resistance of bimaterial interfaces, Acta Metall., 37(3), pp. 909-916, DOI: 10.1016/0001-6160(89)90017-5.

[4] Shah, S.P., Swartz, S.E., and Ouyang, C. (1995).Fracture Mechanics and Compressive Failure. Fracture Mechanics of Concrete: Applications of Fracture Mechanics to Concrete, Rock and Other Quasi-Brittle Materials, Wiley, pp.

[5] Brighenti, R., Carpinteri, A., and Spagnoli, A. (2014). Influence of material microvoids and heterogeneities on fatigue crack propagation, Acta Mech., 225(11), pp. 3123-3135, DOI: 10.1007/s00707-014-1111-7.

[6] Bian, L.-C., Fawaz, Z., and Behdinan, K. (2006). A mixed mode crack growth model taking account of fracture surface contact and friction, Int. J. Fract., 139(1), pp. 39-58, DOI: 10.1007/s10704-006-6633-0.

[7] Ballarini, R. and Plesha, M.E. (1987). The effects of crack surface friction and roughness on crack tip stress fields, Int. J. Fract., 34(3), pp. 195-207, DOI: 10.1007/BF00019717.

[8] Plesha, M.E. (1987). Constitutive models for rock discontinuities with dilatancy and surface degradation, Int. J. Numer. Anal. Methods Geomech., 11(4), pp. 345-362, DOI: 10.1002/nag.1610110404.

[9] Tong, J., Yates, J.R., and Brown, M.W. (1995). A model for sliding mode crack closure part II: mixed mode I and II loading and application, Eng. Fract. Mech., 52(4), pp. 613-623, DOI: 10.1016/0013-7944(95)00045-W.

[10] Carpinteri, A., Spagnoli, A., Terzano, M., and Vantadori, S. (2017). Fracture toughness of rough and frictional cracks emanating from a re-entrant corner, Frat. Ed Integrita Strutt., 41, pp. 175-182, DOI: 10.3221/IGF-ESIS.41.24.

[11] Hills, D.A., Kelly, P.A., Dai, D.N., and Korsunsky, A.M. (2013). Solution of Crack Problems: The Distributed Dislocation Technique, Springer Netherlands.

[12] Spagnoli, A., Carpinteri, A., and Terzano, M. (2018). Near-tip stress fields of rough and frictional cracks under mixedmode loading, Fatigue Fract. Eng. Mater. Struct., 41(4), pp. 2099-2109, DOI: 10.1111/ffe.12765.

[13] Carpinteri, A. (1994). Fractal nature of material microstructure and size effects on apparent mechanical properties, Mech. Mater., 18(2), pp. 89-101, DOI: 10.1016/0167-6636(94)00008-5.

[14] Carpinteri, A. and Spagnoli, A. (2004). A fractal analysis of size effect on fatigue crack growth, Int. J. Fatigue, 26(2), pp. 125-133, DOI: 10.1016/S0142-1123(03)00142-7.

[15] Carpinteri, A., Spagnoli, A., Vantadori, S., and Viappiani, D. (2008). Influence of the crack morphology on the fatigue crack growth rate: A continuously-kinked crack model based on fractals, Eng. Fract. Mech., 75, pp. 579-589, DOI: 10.1016/j.engfracmech.2007.05.007.

[16] Carpinteri, A., Spagnoli, A., and Vantadori, S. (2010). A multifractal analysis of fatigue crack growth and its application to concrete, Eng. Fract. Mech., 77(6), pp. 974-984, DOI: 10.1016/j.engfracmech.2010.01.019.

[17] Cordisco, F.A., Zavattieri, P.D., Hector, L.G., and Carlson, B.E. (2016). Mode I fracture along adhesively bonded sinusoidal interfaces, Int. J. Solids Struct., 83, pp. 45-64, DOI: 10.1016/j.ijsolstr.2015.12.028.

[18] Shah, S.P. and Ouyang, C. (1994). Fracture mechanics for failure of concrete, Annu. Rev. Mater. Sci., 24(1), pp. 293320.

[19] Xie, H.B., Jiang, Z.Y., and Yuen, W.Y.D. (2011). Analysis of friction and surface roughness effects on edge crack evolution of thin strip during cold rolling, Tribol. Int., 44(9), pp. 971-979, DOI: 10.1016/j.triboint.2011.03.029. 